\title{
Beyond Strömgren Spheres and Wind-Blown Bubbles: An Observational Perspective on H II Region Feedback
}

\author{
Matthew S. Povich*i \\ Penn State \\ E-mail: povicheastro.psu.edu
}

\begin{abstract}
Massive stars produce copious quantities of ultraviolet radiation beyond the Lyman limit, photoionizing the interstellar medium (ISM) and producing H II regions. As strong sources of recombination- and forbidden-line emission, infrared continuum, and thermal (free-free) radio continuum, H II regions serve as readily-observable beacons of massive star formation in the Milky Way and external galaxies. Along with supernovae, H II regions are dominant sources of feedback in star-forming galaxies, injecting radiative and mechanical luminosity into the ISM. $\mathrm{H}$ II regions may prove more important than supernovae as triggers of star formation through localized compression of cold cloud cores. In this review, I give a broad overview of the structure and time-evolution of $\mathrm{H}$ II regions, emphasizing complications to the theoretical picture revealed by multiwavelength observations. I discuss a recent controversy surrounding the dominant feedback mechanism in 30 Doradus, the most luminous H II region in the Local Group. I summarize the first results from the Milky Way Project (MWP), which has produced a new catalog of several thousand candidate Galactic H II regions by enlisting >35,000 "citizen scientists" to search Spitzer Space Telescope survey images for bubble-shaped structures. The MWP and similar large catalogs enable empirical studies of Galactic $\mathrm{H}$ II region evolution across the full range of luminosities and statistical studies of triggered star formation.
\end{abstract}

Frank N. Bash Symposium 2011: New Horizons in Astronomy

October 9-11, 2011

Austin, Texas, USA

\footnotetext{
* Speaker.

${ }^{\dagger}$ NSF Astronomy and Astrophysics Postdoctoral Fellow.
} 


\section{Introduction: The Interstellar Medium Out of Balance}

Most students of astronomy will encounter a course on the physics of the interstellar medium (ISM; the gas and dust occupying the space between the stars) at some point in their undergraduate or post-graduate studies. Hence the concept that the ISM exists in several distinct "phases," in rough pressure equilibrium with each other, may be familiar to most readers. Physical quantities characterizing the major phases of ISM gas in the Milky Way are summarized in Table 1, compiled from the textbooks by Tielens [1] and Draine [2]. The coexistence of gas at such dramatically different densities, temperatures, and ionization fractions in the same galaxy is explained by the different filling factors of each phase. While the precise values for the filling factors remain poorlymeasured and controversial, the basic picture that the colder, denser phases of the ISM exists as smaller clouds within the more diffuse, warm/hot phases, is well established. $\mathrm{H}$ II regions, localized regions of photo-ionized gas produced by hot, massive, OB-type stars, occupy a negligible fraction of the ISM volume, hence perhaps H II regions ought not to be regarded as a proper ISM phase at all. However, massive stars form in the densest regions of cold, molecular clouds, and as their far-ultraviolet (UV) radiation first photo-dissociates molecules and then photo-ionizes atoms, the multi-phase physics of the ISM can be studied within a single, small volume.

\begin{tabular}{lccccc}
\hline \hline Phase & $\begin{array}{c}\text { Density } \\
\left(\mathrm{cm}^{-3}\right)\end{array}$ & $\begin{array}{c}T \\
(\mathrm{~K})\end{array}$ & $\begin{array}{c}\text { Total Mass } \\
\left(10^{9} \mathrm{M}_{\odot}\right)\end{array}$ & $\begin{array}{c}\text { Scaleheight } \\
(\mathrm{pc})\end{array}$ & $\begin{array}{c}\text { Filling } \\
\text { factor }\end{array}$ \\
\hline Hot ionized medium & $\sim 0.004$ & $\sim 10^{6}$ & - & 3000 & $\sim 0.5$ \\
Warm neutral medium & $0.5-0.6$ & 8000 & 2.8 & $\sim 300$ & $\sim 0.4$ \\
Warm ionized medium & $0.1-0.3$ & $\sim 5000$ & 1.0 & 900 & $\sim 0.1$ \\
Cold neutral medium & $30-50$ & $80-100$ & 2.2 & 100 & $\sim 0.01$ \\
Molecular Clouds & $10^{2}-10^{6}$ & $10-50$ & 1.3 & 75 & $\sim 10^{-4}$ \\
\hline H II Regions & $1-10^{4}$ & $10^{4}$ & 0.05 & 70 & - \\
\hline
\end{tabular}

Table 1: Phases of ISM Gas in the Milky Way

In one of the immortalized insights of early modern astrophysics, Strömgren [3] realized that the mean free path in neutral hydrogen of UV photons beyond the Lyman limit $(\lambda<912 \AA)$ is negligibly small compared to the size of the ionized hydrogen region produced by a hot star. $\mathrm{H}$ II regions therefore have sharp boundaries, or I-fronts, where the recombination rate balances the ionization rate. For a single star in an ambient medium of constant density, this boundary is defined as the Strömgren radius,

$$
R_{S 0}=\left(\frac{3 Q_{0}}{4 \pi n_{H}^{2} a_{B}}\right)^{1 / 3},
$$

where $Q_{0}$ is the ionizing photon rate (which depends on the star or stars responsible for the $\mathrm{H}$ II region), $n_{H}$ is the hydrogen gas density, and $a_{B}$ is the Case B recombination coefficient [4]. Generally speaking, main-sequence or giant stars earlier than B3 emit sufficient $Q_{0}$ to produce observable Galactic H II regions.

Because the ionizing stars provide an internal source of radiative and mechanical luminosity, $\mathrm{H}$ II regions rapidly become overpressured compared to the ambient ISM and expand. If the dom- 
inant source of pressure is collisional heating of gas by free electrons, then the time-evolution of the expansion follows the simple analytic relation from Lyman Spitzer's classic ISM text [4]:

$$
R_{S}(t)=\left(1+\frac{7}{4} \frac{c_{S 2} t}{R_{S 0}}\right)^{4 / 7},
$$

where $c_{s 2} \sim 10 \mathrm{~km} \mathrm{~s}^{-1}$ is the sound speed in the ionized gas. The expansion velocity obtained by differentiating this relation can exceed the (significantly lower) sound speed in the ambient medium, hence expanding I-fronts often become shock waves. If the ambient medium is molecular, UV photons emerging from the $\mathrm{H}$ II region destroy the molecules, creating a photodissociation region (PDR) around the $\mathrm{H}$ II region.

Early $\mathrm{O}$ stars and $\mathrm{OB}$ giants drive powerful winds that fundamentally alter the structure of more luminous H II regions. Castor et al. [5] and Weaver et al. [6] provided analytical models for wind-blown bubbles produced by isolated, hot stars, and numerous authors have subsequently refined these models using a variety of semi-analytical and numerical techniques [7-11]. The basic, "onion-layer" structure of a wind-blown bubble is illustrated in Figure 1. The highly supersonic (1000-2000 $\mathrm{km} \mathrm{s}^{-1}$ ) stellar wind flows freely outward for a short distance $R_{W}$ from the star before it is shocked, producing a bubble of very hot, ionized gas. The "classical" H II region collapses into a photoionized shell of gas, (imperfectly) separated from the hot gas zone by a contact discontinuity at $R_{C}$. The I-front at $R_{I F}$ still represents the outer boundary of the $\mathrm{H}$ II region.

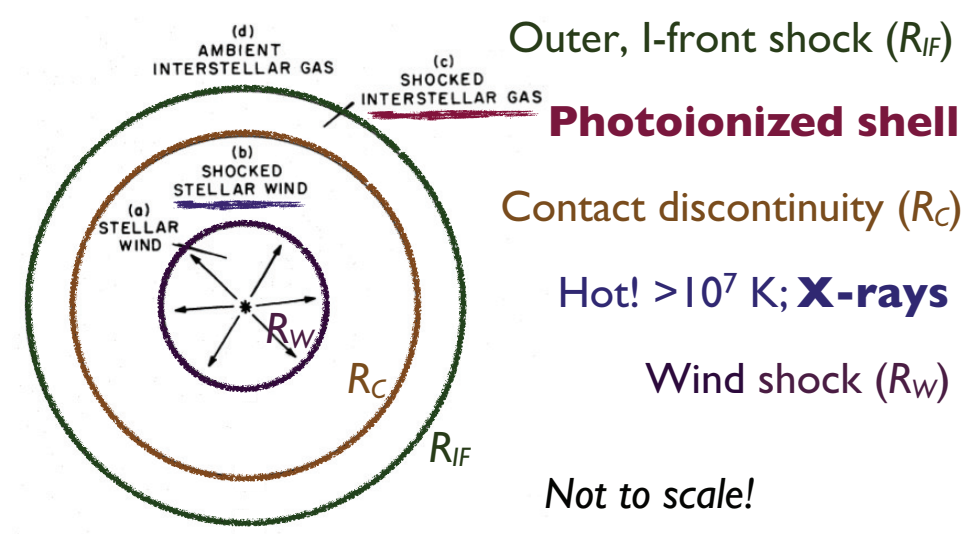

Figure 1: Anatomy of a wind-blown bubble, adapted from Weaver et al. [6]

Many complications separate the ideal models of Strömgren spheres and wind-blown bubbles from reality. The ISM is clumpy, hence the ambient medium surrounding an $\mathrm{H}$ II region is never uniform. Massive stars tend to form in clusters, hence multiple stars often contribute to the ionization of a single $\mathrm{H}$ II region. The ambient ISM is generally in motion with respect to the ionizing star(s), and stellar winds need not be spherically symmetric. Dust mixed with gas in H II regions enables dramatic, radiative cooling [12], and magnetic fields threading through the clouds contribute anisotropic pressure support [13]. Turbulence provides additional pressure and facilitates mixing at the interfaces between gas layers. Early models neglected completely the contribution of radiation pressure $[14,15]$. 


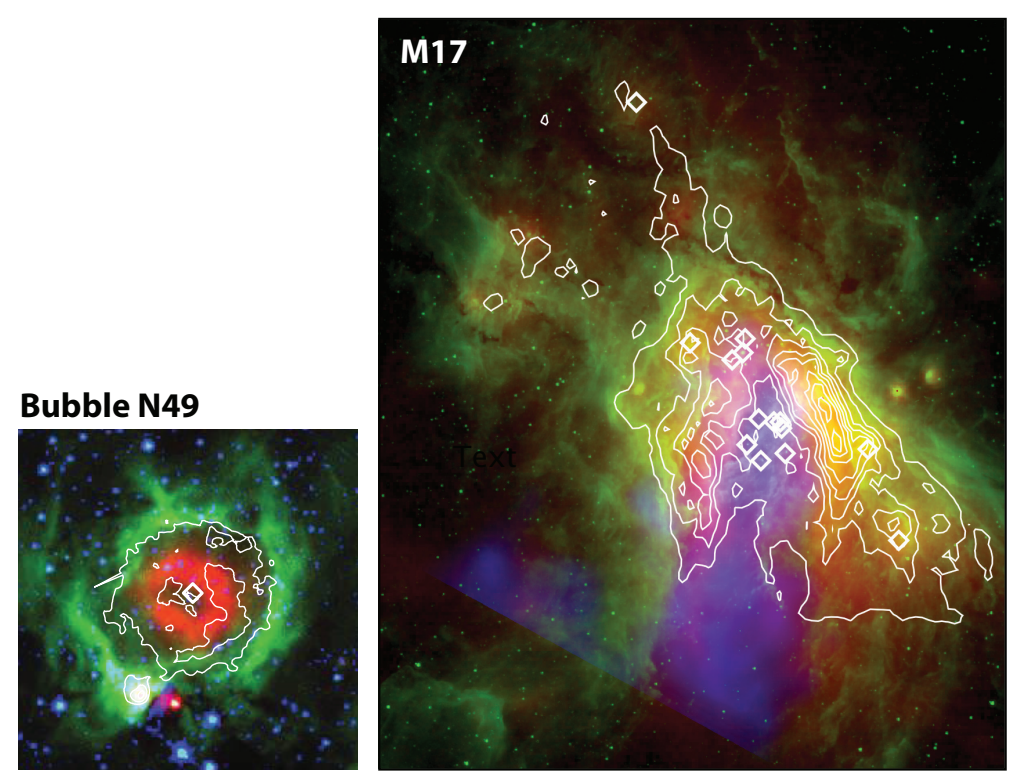

Figure 2: Multiwavelength images of the wind-blown bubble N49 [17] and the giant H II region M17 [18], displayed at approximately the same physical scale. N49 image: red = Spitzer/MIPS $24 \mu \mathrm{m}$, green/blue = Spitzer/IRAC 8.0/4.5 $\mu \mathrm{m}$. M17 image: red $=M S X 21.3 \mu \mathrm{m}$, green $=$ IRAC $5.8 \mu \mathrm{m}$, blue $=$ Chandra soft $(0.5-2 \mathrm{keV})$, diffuse X-rays. In both images, diamonds denote known $\mathrm{O}$ and early $\mathrm{B}$ stars and contours show $20 \mathrm{~cm}$ thermal radio continuum.

\section{Multiwavelength Observations of H II Regions}

In spite of the messy complexity governing the structure of real H II regions, the basic structures predicted by the wind-blown bubble models are identifiable in modern, multiwavelength images. In Figure 2, a prototypical wind-blown bubble ionized by a single $06.5 \mathrm{~V}$ star $\left(Q_{0}=\right.$ $\left.8.5 \times 10^{48} \mathrm{~s}^{-1}\right)[16,17]$ is compared to the giant $\mathrm{H}$ II region M17, ionized by a dozen $\mathrm{O}$ stars, including several O4 V stars $\left(Q_{0}=3 \times 10^{50} \mathrm{~s}^{-1}\right)$ [18]. In $\mathrm{N} 49$, the I-front at $R_{I F}$ is defined by the sharp inner rim of $8.0 \mu \mathrm{m}$ (green) polycyclic aromatic hydrocarbon (PAH) emission from the PDR, neatly encapsulating the photoionized gas shell (contours). Dust mixed with the photoionized gas and heated by radiation from the central star forms a torus of $24 \mu \mathrm{m}$ emission. Everett \& Churchwell [12] found that the lifetime of dust grains within the harsh environment of this $\mathrm{H}$ II region is extremely short, and suggested that dust must be continuously replenished from evaporating dense clumps to produce the observed $24 \mu \mathrm{m}$ emission. Draine [15] demonstrated that radiation pressure can produce evacuated cavities in the centers of $\mathrm{H}$ II regions, but noted that the central hole in N49 is too large to be explained by radiation pressure alone and suggested that the stellar wind also contributes. An interface analogous to the contact discontinuity $R_{C}$ in Figure 1 is likely located within the radio shell/24 $\mu \mathrm{m}$ torus in $\mathrm{N} 49$, hence $R_{C} / R_{I F}<1 / 2$.

Unlike N49, M17 is far from round, yet similar morphological features can be discerned, with one important addition (Figure 2). A spectacular plume of hot, X-ray-emitting plasma (blue) occupies the central cavity of M17 [19]. This X-ray emission provides direct evidence for stellar 
wind shocks. At an absorption-corrected X-ray luminosity $L_{X}=7 \times 10^{34} \mathrm{erg} \mathrm{s}^{-1}$, this plasma is unusually bright in comparison to other $\mathrm{H}$ II regions, but it is fainter than the predictions of wind-blown-bubble theory by more than an order of magnitude [20]. This discrepancy may be explained by collisional interactions with dust grains providing a mechanism for cooling the hot plasma, and/or depressurization of the wind-blown bubble where the plasma is not completely confined by the nebula. Either interpretation implies that the contact discontinuity in Figure 1 does not effectively separate the photoionzed gas/dusty shell from the hot gas bubble in M17. Again assuming $R_{C}$ falls at the inner edge of the photoionized shell and heated dust emission, $R_{C} / R_{I F} \approx 1 / 2$ in M17. The inner cavity in M17 is clearly larger (both in absolute volume and as a fraction of the H II region volume) in comparison to that of N49. The photoionized shell in M17 is supported by a combination of radiation pressure and hot gas pressure [13].

Although N49 and M17 are representative of a range of Galactic H II regions where stellar winds play an important role, on the Galactic scale feedback is dominated by the most luminous, starburst regions. In Figure 3, a Galactic starburst region, W43, is compared to 30 Doradus in the Large Magellanic Cloud (LMC), the most luminous H II region in the Local Group $\left(Q_{0}=4.2 \times 10^{51}\right.$ $\mathrm{s}^{-1}$; [21]). Mid-infrared (IR) images of the starburst H II regions reveal layered bubble morphologies that are remarkably similar between the two regions. The large bubble lobes shown in each panel of Figure 3 are part of larger H II region complexes, with the ionizing clusters partially (in 30 Dor) or completely (W43) obscured by dense, foreground filaments of bright, mid-IR emission. In the 30 Dor image, the PDRs appear pink and the photo-ionized shells green, while the W43 image matches the color-code of Figure 2, in which the PDRs appear yellow-green and dust mixed within photoionized shell appears red. Here it is most appropriate to describe the photoionized gas struc-
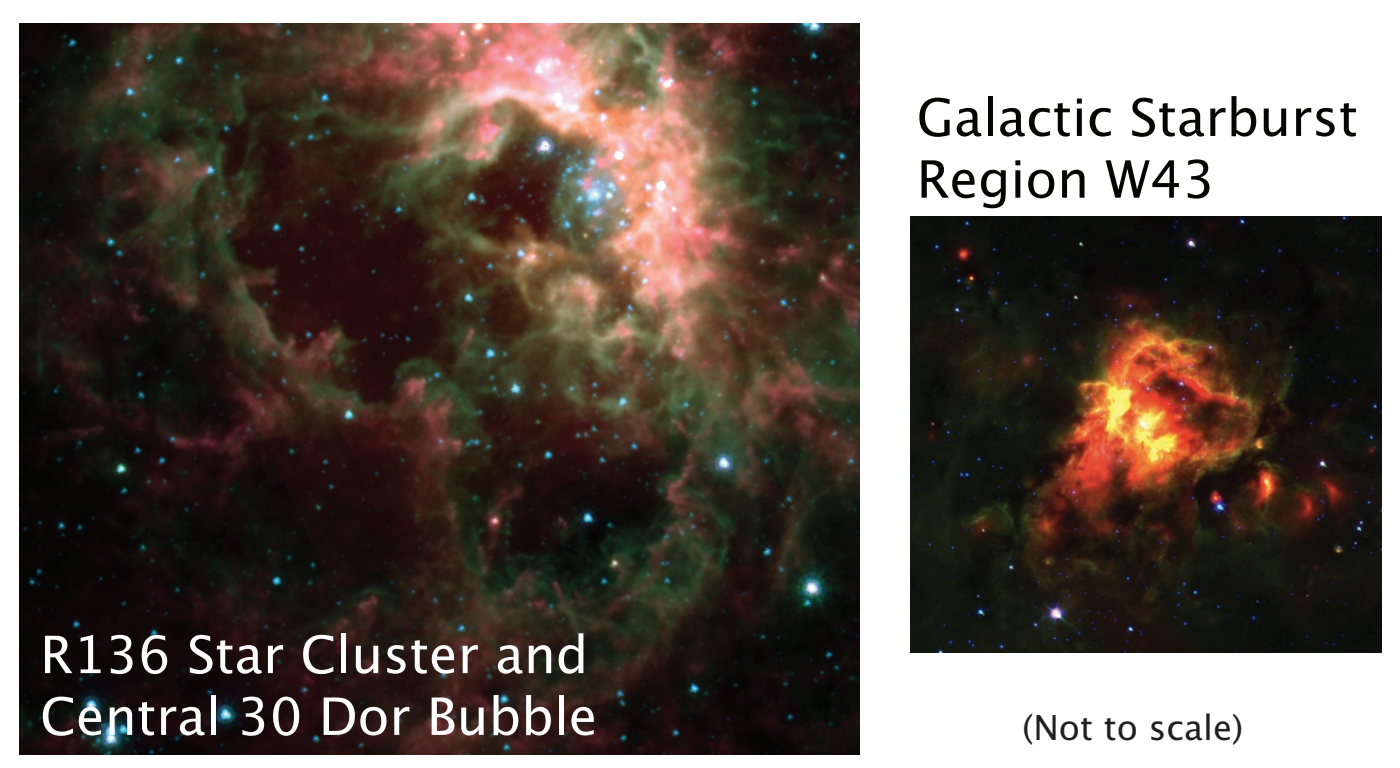

(Not to scale)

Figure 3: Spitzer mid-IR images of two starburst H II regions. Left: Image of 30 Dor in the Large Magellanic Cloud, red $=8.0 \mu \mathrm{m}$, green $=4.5 \mu \mathrm{m}$, blue $=3.6 \mu \mathrm{m}$. Right: Image of $\mathrm{W} 43$, red $=24 \mu \mathrm{m}$, green $=8.0 \mu \mathrm{m}$, blue $=4.5 \mu \mathrm{m}$. 
tures as shells, occupying thin layers just interior to the PDRs, with $R_{C} \approx R_{I F}$. Indeed, the bubbles in 30 Dor are known to be filled with hot, X-ray-emitting plasma [22], and similar plasma would likely be found in W43 if comparable Chandra X-ray Observatory observations were obtained.

Comparing Figures 2 and 3, a trend becomes apparent: as the luminosity of the ionizing cluster increases, so does $R_{C} / R_{I F}$, the size of the central cavity relative to the overall size of the $\mathrm{H}$ II region. We may extend this trend down to low-luminosity $\mathrm{H}$ II regions ionized by late $\mathrm{O}$ or early B stars, for which the central cavity disappears entirely $\left(R_{C}=0\right)$. It will be useful to bear this trend in mind when considering the controversial issue of precisely which feedback mechanism, hot gas pressure or direct radiation pressure, dominates $\mathrm{H}$ II region structure at the high-luminosity extreme.

\section{A Rumble in the Tarantula: What is the Dominant Feedback Mechanism in $\mathbf{3 0}$ Doradus?}

Thanks to its status as the most luminous H II region in the Local Group and its location in the low-metallicity environment of the LMC, 30 Dor (popularly known as the Tarantula Nebula) has long received intense observational scrutiny. At $d=50 \mathrm{kpc}, 30$ Dor is the best nearby laboratory for studying the physical conditions that prevailed in the unresolvable, high-redshift star-forming regions that dominated the major cosmological epoch of galaxy-building [21]. Recently, Lopez et al. [23, hereafter L11] and Pellegrini et al. [21, hereafter P11] carried out independent, parallel studies of the feedback processes shaping 30 Dor. Using fundamentally different approaches to interpreting multiwavelength datasets, these authors reached diametrically opposed conclusions; L11 reported that direct stellar radiation pressure dominates the interior of the $\mathrm{H}$ II region, while P11 argued that the pressure of the hot, X-ray-emitting plasma shapes the large-scale structure and dynamics. This disagreement is rooted in the different definitions of radiation pressure and the different assumed nebular geometries used in the two studies.

L11 used the simplest definition of direct radiation pressure,

$$
P_{\mathrm{dir}}=\sum \frac{L_{\mathrm{bol}}}{4 \pi r^{2} c}
$$

(their equation 1), where $L_{\mathrm{bol}}$ is the bolometric luminosity of each star and $r$ is the distance traveled by the starlight to reach a given point in the nebula, deprojected assuming a spherical geometry. $P_{\text {dir }}$ declines sharply with distance from the central star cluster, R136 (note that this expression diverges for $r=0$ ).

By contrast, P11 constructed a non-symmetric, cavity model (based on the central region of 30 Dor shown in Figure 3) for the nebular geometry and used photoionization models to calculate the density of $\mathrm{H}$ atoms $n_{H}$ and hence the ionization parameter $U$ at each position,

$$
U=\frac{Q_{0}}{4 \pi r^{2} c n_{H}} .
$$

The divergent behavior of this expression is avoided by implementing the cavity model, in which the ionized gas is confined to shell structures near the I-fronts, and $U$ is not calculated for the cavity interiors, where $r^{2} n_{H} \rightarrow 0$. P11 then approximated the pressure exerted on the observed ionized gas by starlight in terms of the ionization parameter as

$$
P_{\text {stars }}=U n_{H}\langle h v\rangle \frac{L_{\mathrm{bol}}}{L_{0}}
$$


where $L_{\mathrm{bol}}$ and $L_{0}$ are the total bolometric and ionizing photon luminosity for all stars (assumed to be centered at R136) and $\langle h v\rangle \sim 20 \mathrm{eV}$ is average energy per ionizing photon (their equation 8).

Although Equations 3.3 and 3.2 can be combined and trivially reduced to Equation 3.1, doing so hides the ambiguous role of radiation pressure in regions where $n_{H}$ vanishes. $P_{\text {stars }}$ as definited by P11 represents the momentum imparted to the observed nebular gas. L11 acknowledge this alternative definition of radiation pressure, but claim that "it is necessary to characterize $P_{\text {dir }}$ as the energy density of the radiation field, since that definition reflects the total energy and momentum budget available to drive motion." This definition implies that the luminosity emitted by the OB stars could impart momentum with $100 \%$ efficiency everywhere in the nebula at once, an ideal case that could never occur in a real $\mathrm{H}$ II region. The justification brings to mind the old philosophical thought experiment about whether a tree falling in a forest makes a sound if there is no one around to hear it. If one dropped a cloud of dense, neutral gas close to the R136 star cluster, it would experience an enormous radiation pressure. But there are no dense clouds of neutral gas within the radiation-pressure-dominated region identified by L11. Instead, the interior structure of 30 Dor consists of evacuated cavities filled with hot, highly ionized plasma [22], and pressure from either the hot, X-ray plasma or the warm, photoionized gas can exceed the radiation pressure on the cavity walls (L11, P11). Radiation pressure could have dominated the expansion of these large cavities in the past, when they were smaller (P11). It is difficult to answer this question definitively because the hot gas pressure in 30 Dor remains uncertain.

Both L11 and P11 reported values for the hot gas pressure in 30 Dor. The pressure of the $\mathrm{X}$-ray-emitting plasma can be calculated as

$$
P_{X}=1.9 n_{X} k T_{X}
$$

where $n_{X}$ and $T_{X}$ are the density and temperature of the X-ray-emitting plasma. P11 used the results of spectral fits by Townsley et al. [22] while L11 performed their own spectral fitting to the same archival data plus a newer, 90-ks Chandra observation of 30 Dor [24]. It is difficult to derive $n_{X}$ accurately from the emission measure returned by spectral fitting because it depends strongly on the assumed geometry of the X-ray bubbles. L11 treated 30 Dor as a "beach ball" with a global, spherical geometry, which effectively minimizes $n_{X}$. P11 treated 30 Dor as a "bunch of grapes," assuming a spherical geometry for each smaller, diffuse X-ray region identified by Townsley et al. [22], which yields higher $n_{X}$. Spectral modeling of diffuse emission structures in regions like 30 Dor is a tremendously complicated task [20], as any given sightline will contain plasma from multiple origins, including both stellar wind shocks and supernova, at a variety of temperatures, densities, and compositions. To illustrate the pitfalls of over-interpreting these data, I note that different approaches to the spectral fitting of the the global, diffuse X-ray emission of 30 Dor yield significantly different results. L11 fit a single-temperature plasma model and reported $k T_{X}=0.64_{-0.02}^{+0.03} \mathrm{keV}$ and absorption-corrected $L_{X}=4.5 \times 10^{36} \mathrm{erg} \mathrm{s}^{-1}$. In contrast, the most recent spectral fits by L. K. Townsley (private communication) employ 3 plasma components (plus numerous gaussian profiles to fit unidentified emission lines) ranging from $0.3-0.8 \mathrm{keV}$ and yield $L_{X}=1.2-1.9 \times 10^{37} \mathrm{erg} \mathrm{s}^{-1}$. It is particularly difficult to discern whether regions of 30 Dor that appear X-ray dark represent the boundaries of confined, hot plasma bubbles, or whether the plasma extends behind foreground material that absorbs the soft X-rays. The existing $114 \mathrm{ks}$ 
combined Chandra integration represents a very shallow observation at the distance of 30 Dor when compared to observations of Galactic H II regions.

The story of 30 Dor has a moral; given the complexity and ambiguity involved in interpreting the multiwavelength data on this resolved starburst region, investigators wishing to extend these results to draw conclusions about feedback mechanisms shaping unresolved regions at cosmological distances do so at their own risk.

\section{The Milky Way Project H II Region Catalog}

Strong empirical constraints on the time-evolution of feedback-driven $\mathrm{H}$ II regions require the comparative study of large observational samples of $\mathrm{H}$ II regions. The wealth of new IR imaging data provided by the Spitzer Galactic Legacy Infrared Mid-Plane Extraordinaire (GLIMPSE) [25] and subsequent high-resolution surveys of the Milky Way allow us to penetrate the obscuring veil of dust in the Galactic plane, revealing the structure of $\mathrm{H}$ II regions and PDRs in unprecedented detail. Using the GLIMPSE images, Churchwell et al. [16, 26] cataloged nearly 600 IR bubbles, ring and arc-shaped structures apparent in $8 \mu \mathrm{m}$ PAH emission. The majority of these structures are PDRs surrounding $\mathrm{H}$ II regions, from energetic, wind-blown bubbles to low-luminosity nebulae surrounding B-type stars.

Over the past year, $>35,000$ internet users from around the world have been searching for bubbles in Spitzer survey images of the Galactic plane as part of the Milky Way Project (MWP; http://www.milkywayproject.org), a recent installment in the Zooniverse, the premier series of online "citizen science" projects (http://www.zooniverze.org). Upon creating a Zooniverse account and logging into milkywayproject.org, MWP volunteers are presented with a random image and instructed to identify and fit structures within that image that resemble bubble rims (PDRs resembling the regions in Figure 2) with elliptical annuli (or mark the locations of bubbles that are barely resolved with boxes). By carefully combining the results from many individuals for each part of the sky, the MWP simultaneously leverages the superior pattern-recognition skills of the human eye-brain system and takes advantage of the "wisdom of crowds." The MWP First Data Release presents a catalog of 5,106 $\mathrm{H}$ II regions, representing an order of magnitude improvement in completeness compared to previous catalogs [27].

The MWP bubbles catalog will facilitate the study of triggered star formation on the Galactic scale. The idea that feedback from expanding H II regions can exert external pressure on cold, molecular cloud cores, resulting in self-propagating, sequential massive star formation has been around for decades [28-31] but recently has seen a resurgence of observational and theoretical interest, motivated in large part by the identification of numerous instances of small bubbles, young stellar objects, masers, and other observational signposts of recent or ongoing star formation near the rims of GLIMPSE bubbles (N49 in Figure 2 provides an example, with two luminous young stellar objects and an ultracompact $\mathrm{H}$ II region visible on the lower rim of the bubble [17]). To date, most studies of triggered star formation have focused on individual, "best-case" regions, very round bubbles with prominent sub-clusters or smaller bubbles on their rims [32-34]. In spite of the long-standing, widely popular idea that supernovae trigger star formation, far fewer candidate triggering sites have been identified near supernova remnants than near $\mathrm{H}$ II regions. 
To establish that triggering merits investigation as an important mode of star formation as opposed to an astrophysical curiosity, the prevalence of triggering sites must be established statistically, and in an unbiased fashion, among representative samples of $\mathrm{H}$ II regions. Both the GLIMPSE and MWP bubbles catalogs include flags for hierarchical structure, identifying smaller bubbles that could be the "daughters" of larger, "parent" bubbles. Among the MWP bubbles, 29\% are members of hierarchies [27]. Triggered star formation need not produce observable daughter bubbles if the second generation of stars is too young to have produced H II regions. Thompson et al. [35] recently found a strong correlation between young stellar objects identified as luminous mid-IR point sources and the rims of bubbles from the Churchwell et al. [16] catalog. Corroboration of this correlation using the more complete sample of bubbles from the MWP would greatly strengthen the case that triggering is a prevalent mode of Galactic star formation.

\section{Summary}

In this review, I have given a brief update on recent, multiwavelength observations, particularly in the mid-IR and X-rays, that have revealed wind-blown and radiation-dominated $\mathrm{H}$ II region structures. Observations corroborate the basic predictions of $\mathrm{H}$ II region theory but reveal important differences, too. Theory still struggles to explain both the existence of dust within energetic $\mathrm{H}$ II regions and its effects on $\mathrm{H}$ II region structure. X-rays from hot, wind-shocked plasma are frequently observed to fill large, central cavities in giant $\mathrm{H}$ II regions, but the X-ray luminosity is more than an order of magnitude lower than predicted by wind-blown bubble theory.

The size of the central cavities as a fraction of $\mathrm{H}$ II region volume appears to increase with increasing ionizing luminosity (Figures 2 and 3). This trend puts dust at larger distances from the ionizing stars in starburst regions, which reduces the effective temperature of the dust compared to static or thermal pressure-dominated H II region models. Single-band IR diagnostics of extragalactic star formation rates (e.g. [36]) have become increasingly popular in the era of large, high-resolution surveys from Spitzer and Herschel. Because the effective dust temperature sets the shape of the IR spectral energy distribution in unresolved regions, feedback must be taken into account when calibrating the IR diagnostics.

Feedback in large, starburst regions like 30 Dor presents a particularly complicated problem. It would be premature to assume that a single source of feedback, radiation pressure (L11), dominates such regions until we achieve a better understanding of the contributions from massive star winds and supernovae.

$\mathrm{H}$ II regions are among the most beautiful objects appearing in astronomical images. The strong aesthetic appeal of these images helped to motivate MWP volunteers, the vast majority of whom were not professional scientists, to spend tens of thousands of person-hours finding and measuring several thousand H II regions in the form of IR bubbles. The large MWP database [27] provides an unmatched resource for statistical studies of $\mathrm{H}$ II region evolution and star formation triggered by massive star feedback. I expect that the MWP will spawn many follow-up investigations in the coming years, involving both professional researchers and citizen scientists. 


\section{Acknowledgments}

I thank the organizers of the Frank N. Bash Symposium 2011 for their invitation to speak and to contribute to these proceedings. L. K. Townsley and E. W. Pellegrini provided helpful conversations and insights that greatly improved this contribution. I gratefully acknowledge support from a National Science Foundation Astronomy \& Astrophysics Postdoctoral Fellowship under award AST-0901646.

\section{References}

[1] Tielens, A. G. G. M., The Physics and Chemistry of the Interstellar Medium, Cambridge University Press, 2005

[2] Draine, B. T., Physics of the Interstellar and Intergalactic Medium, Princeton University Press, 2011

[3] Strömgren, B., The Physical State of Interstellar Hydrogen., 1939, ApJ, 89, 526

[4] Spitzer, L., Physical processes in the interstellar medium, Wiley-Interscience, New York, 1978

[5] Castor, J., R. McCray, \& R. Weaver, Interstellar bubbles, 1975, ApJ, 200, L107

[6] Weaver, R., et al., Interstellar bubbles. II - Structure and evolution, 1977, ApJ, 218, 377

[7] McKee, C. F., D. van Buren, \& B. Lazareff, Photoionized stellar wind bubbles in a cloudy medium, 1984, ApJ, 278, L115

[8] Koo, B.-C. \& C. F. McKee, Dynamics of wind bubbles and superbubbles. I - Slow winds and fast winds. II - Analytic theory, 1992, ApJ, 388, 93

[9] Capriotti, E. R. \& J. F. Kozminski, Relative Effects of Ionizing Radiation and Winds from O-Type Stars on the Structure and Dynamics of H II Regions, 2001, PASP, 113, 677

[10] Freyer, T., G. Hensler, \& H. W. Yorke, Massive Stars and the Energy Balance of the Interstellar Medium. I. The Impact of an Isolated $60 M_{\odot}$ Star, 2003, ApJ, 594, 888

[11] Harper-Clark, E. \& N. Murray, One-Dimensional Dynamical Models of the Carina Nebula Bubble, 2009, ApJ, 693, 1696

[12] Everett, J. E. \& E. Churchwell, Dusty Wind-blown Bubbles, 2010, ApJ, 713, 592

[13] Pellegrini, E. W., et al., A Magnetically Supported Photodissociation Region in M17, 2007, ApJ, 658, 1119

[14] Krumholz, M. R. \& C. D. Matzner, The Dynamics of Radiation-pressure-dominated H II Regions, 2009, ApJ, 703, 1352

[15] Draine, B. T., On Radiation Pressure in Static, Dusty H II Regions, 2011, ApJ, 732, 100

[16] Churchwell, E., et al., The Bubbling Galactic Disk, 2006, ApJ, 649, 759

[17] Watson, C., et al., Infrared Dust Bubbles: Probing the Detailed Structure and Young Massive Stellar Populations of Galactic H II Regions, 2008, ApJ, 681, 1341

[18] Povich, M. S., et al., A Multiwavelength Study of M17: The Spectral Energy Distribution and PAH Emission Morphology of a Massive Star Formation Region, 2007, ApJ, 660, 346

[19] Townsley, L. K., et al., 10 MK Gas in M17 and the Rosette Nebula: X-Ray Flows in Galactic H II Regions, 2003, ApJ, 593, 874 
[20] Townsley, L. K., et al., The Integrated Diffuse X-ray Emission of the Carina Nebula Compared to Other Massive Star-forming Regions, 2011, ApJS, 194, 16

[21] Pellegrini, E. W., J. A. Baldwin, \& G. J. Ferland, Structure and Feedback in 30 Doradus. II. Structure and Chemical Abundances, 2011, ApJ, 738, 34

[22] Townsley, L. K., et al., A Chandra ACIS Study of 30 Doradus. I. Superbubbles and Supernova Remnants, 2006, AJ, 131, 2140

[23] Lopez, L. A., et al., What Drives the Expansion of Giant H II Regions?: A Study of Stellar Feedback in 30 Doradus, 2011, ApJ, 731, 91

[24] Townsley, L. K., Diffuse X-ray Structures in Massive Star-forming Regions, 2009, AIPC, 1156, 225

[25] Churchwell, E., et al., The Spitzer/GLIMPSE Surveys: A New View of the Milky Way, 2009, PASP, 121, 213

[26] Churchwell, E., et al., The Bubbling Galactic Disk. II. The Inner 20, 2007, ApJ, 670, 428

[27] Simpson, R. J., et al., The Milky Way Project First Data Release: A Bubblier Galactic Disk, 2012, $M N R A S$, in press [arXiv:1201.6357]

[28] Elmegreen, B. G. \& C. J. Lada, Sequential formation of subgroups in OB associations, 1977, ApJ, 214,725

[29] Sandford, M. T., II, R. W. Whitaker, \& R. I. Klein, Radiation-driven implosions in molecular clouds, 1982, ApJ, 260, 183

[30] Bertoldi, F., The photoevaporation of interstellar clouds. I - Radiation-driven implosion, 1989, ApJ, 346,735

[31] Whitworth, A. P., et al., The Preferential Formation of High-Mass Stars in Shocked Interstellar Gas Layers, 1994, MNRAS, 268, 291

[32] Deharveng, L., A. Zavagno, \& J. Caplan, Triggered massive-star formation on the borders of Galactic H II regions. I. A search for “collect and collapse” candidates, 2005, A\&A, 433, 565

[33] Zavagno, A., et al., Triggered massive-star formation on the borders of Galactic H II regions. II. Evidence for the collect and collapse process around RCW 79, 2006, $A \& A, 446,171$

[34] Zavagno, A., et al., Star formation triggered by the Galactic H II region RCW 120. First results from the Herschel Space Observatory, 2010, A\&A, 518, L81

[35] Thompson, M. A., et al., The statistics of triggered star formation: an overdensity of massive YSOs around Spitzer bubbles, 2011, MNRAS, in press [arXiv:1111.0972]

[36] Calzetti, D., et al., The Calibration of Mid-Infrared Star Formation Rate Indicators, 2007, ApJ, 666, 870 